\title{
Coupling between localized resonance and excitation of surface waves in metal hole arrays
}

\author{
Fumiaki Miyamaru $^{1,2, *}$ and Mitsuo Wada Takeda ${ }^{1}$ \\ ${ }^{1}$ Department of Physics, Faculty of Science, Shinshu University, 3-1-1 Asahi, Matsumoto, Nagano 390-8621, Japan \\ ${ }^{2}$ PRESTO, Japan Science and Technology Agency, Aobaku, Sendai 980-8577, Japan
}

(Received 1 March 2009; published 14 April 2009)

\begin{abstract}
We investigate the transition of the dominant resonant transmission mechanism from localized resonance to excitation of surface waves and vice versa in metal hole arrays. The transition occurs continuously with a change in the length of the rectangular holes. We also study the effect of the incident-beam diameter and surface condition on the transmission spectrum. Our experimental results show the important differences between the two mechanisms. They also confirm the theoretical prediction of Bravo-Abad et al. [Phys. Rev. B 76, 241102(R) (2007)].
\end{abstract}

DOI: 10.1103/PhysRevB.79.153405

PACS number(s): 42.79.Dj

In the last few decades, several studies have investigated resonant transmission properties of electromagnetic waves in metal hole arrays (MHAs). In 1967, Ulrich ${ }^{1}$ reported the band-pass transmission characteristics of MHAs and attributed them to the excitation of surface waves (ESWs), known as Zenneck waves, in the radio-frequency region; these waves are analogous to surface-plasmon polaritons (SPPs) in the visible region. ${ }^{2}$ Later, Ebbesen et al. $^{3}$ conducted a study similar to that of Ulrich and demonstrated that subwavelength MHAs exhibit extraordinary transmission characteristics in the visible region. Since then, many studies have been conducted to investigate the mechanism of resonant transmission in MHAs.

It is widely believed that the main mechanism responsible for resonant transmission is ESWs that is based on the interference of multiple electromagnetic waves scattered by periodically arranged holes. ${ }^{4-6}$ Klien and co-workers ${ }^{7-17}$ have experimentally found that the hole shape also has a strong effect on the transmission characteristics of MHAs; this effect is known as localized resonance, and it has been investigated extensively. Ruan et al. ${ }^{9}$ have calculated the transmission spectra of periodically arranged and randomly distributed rectangular holes and found a transmission peak whose frequency is almost independent of the period. Very recently, some studies have investigated the effects of hole size and aspect ratio on transmission characteristics. ${ }^{10}$ In particular, Lee et al. ${ }^{11}$ have experimentally demonstrated the effect of the length of randomly arranged rectangular holes on transmission spectra and showed that the resonant peak clearly depends on the hole length; this result is consistent with the theory of half-wavelength resonance. They have also found that the periodic structure enhances the transmission peak of localized resonance. Molen et al. ${ }^{14}$ have reported that the dispersion relation of the peak frequency depends on the aspect ratio of rectangular metal holes.

In this Brief Report, we describe a study conducted to better understand the effect of localized resonance in an MHA. We investigate the transition of the dominant resonant transmission mechanism from localized resonance to ESWs by examining the changes in the transmission spectra that occur when we change the length of the rectangular holes. The measured transmission peak of the MHA shows a redshift as the hole length increases; the incident polarization is perpendicular to the fixed axis of the holes. When the hole length is more than the half-period, the peak frequency is equal to the expected frequency of the half-wavelength resonance of the metal hole. In contrast, when the hole length is less than the half-period, the peak frequency gradually increases to the frequency of ESWs. These results indicate that as the hole length decreases, the dominant resonant transmission mechanism changes from localized resonance to ESWs. The coupling of the two mechanisms occurs when their frequencies are close to each other. Furthermore, the transmission spectra indicate that the surface effect in the case of localized resonance is different from that in the case of ESWs. This result clearly shows that the two mechanisms are different. Our results not only establish the physical significance of the transmission characteristics of MHAs but also provide important information for the development of highsensitivity surface wave sensors using MHAs. ${ }^{18,19}$

We fabricated metal hole arrays in a square lattice on a stainless-steel slab with a thickness of $50 \mu \mathrm{m}$, as shown in the inset of Fig. 1(a). The lattice constant of each MHA was $s=50 \mu \mathrm{m}$. The holes had the same width $w=150 \mu \mathrm{m}$, but different lengths $l=190,210,260,310$, and $450 \mu \mathrm{m}$. We

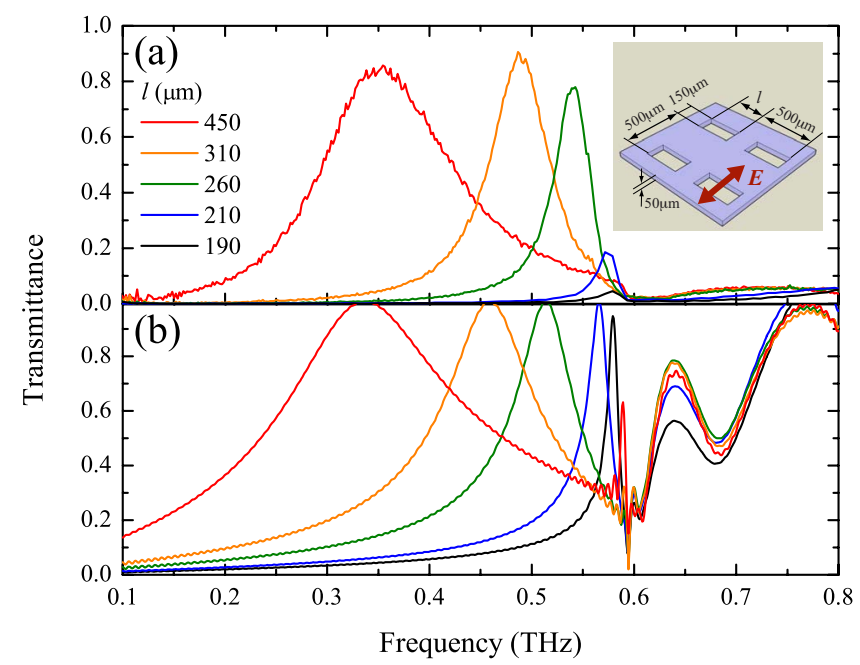

FIG. 1. (Color online) (a) Measured and (b) simulated transmission spectra of metal hole arrays with different hole lengths $l$. The hole width was constant at $w=150 \mu \mathrm{m}$. The inset shows the geometrical parameters of the arrays. 
used a terahertz time-domain spectroscopy system (THzTDS) to measure the transmission spectra of these samples; it allowed us to directly measure the waveform of the terahertz wave in the time domain. We calculated the transmission and phase spectra by Fourier transform of the timedomain waveforms. Details of the THz-TDS system are described in our previous paper. ${ }^{20}$

Figure 1(a) shows the transmission spectra of the MHAs at normal incidence; these spectra were measured by using different values of $l$. Each spectrum exhibits a characteristic transmission peak. The spectrum measured at $l=190 \mu \mathrm{m}$ shows a small transmission peak at around $0.59 \mathrm{THz}$. However, the spectrum measured at $l=450 \mu \mathrm{m}$ shows the same peak at $0.35 \mathrm{THz}$. This observation shows that the peak shifts to lower frequencies as $l$ increases, and it is consistent with the observations of Lee et al. ${ }^{11}$ The transmission intensity $T$ at the peak frequency drastically increases as $l$ increases from $190 \mu \mathrm{m}(T=5 \%)$, and it exceeds $80 \%$ when $l$ exceeds $310 \mu \mathrm{m}$. The bandwidth of the transmission peak also increases gradually with increasing $l$. By using the finitedifference time-domain (FDTD) method, we performed a computational simulation of samples whose geometrical parameters were the same as those in the experiment. The simulation results are shown in Fig. 1(b), and they are in good agreement with the experimental results, except for the peak transmission intensities and spectral shapes above the diffraction frequency $f_{\text {dif }}=0.6 \mathrm{THz}$. The discrepancies above $0.6 \mathrm{THz}$ were attributed to the periodic boundary condition used in our FDTD calculations. The simulated transmission spectra show the effects of higher-order diffracted waves. In contrast, the experimental spectra show the effects of zeroorder electromagnetic waves, because only these waves were measured in the experiment. Hence, because higher-order diffracted waves were detected during the simulation, the artifact appears above the diffraction frequency in the simulated transmission spectra.

In order to better understand the dominant resonant transmission mechanism at each value of $l$, we plot the peak frequency of the transmission spectra as a function of $1 / l$, as shown in Fig. 2. The solid line indicates the expected frequency of localized resonance $\left(\nu_{\text {loc }}\right)$ calculated from the theory of half-wavelength resonance $\left(\nu_{\text {loc }}=c / 2 l\right.$, where $c$ is the speed of light in vacuum). Here, only half-wavelength resonance is taken into account as a mechanism of localized resonance. By considering that the metal thickness $t$ used in our experiment is $50 \mu \mathrm{m}$, we can disregard waveguide resonance, ${ }^{21,22}$ which is caused by Fabry-Pérot resonance between the front and back sides of the metal holes, as a mechanism of localized resonance. The peak frequencies of the measured transmission spectra are indicated by open circles. When $l$ is greater than the half-period $(s / 2$ $=250 \mu \mathrm{m})$, the measured peak frequency is found to be in good agreement with the theoretical frequency of localized resonance. When $l$ is less than $s / 2$, the measured peak frequency deviates from the theoretical frequency of localized resonance and approaches the frequency of ESWs (dotted line), which was calculated from the lattice period $c / s$ and found to be independent of $l$. This result clearly shows that as $l$ decreases, the dominant resonant transmission mechanism changes from localized resonance to ESWs. An inter-

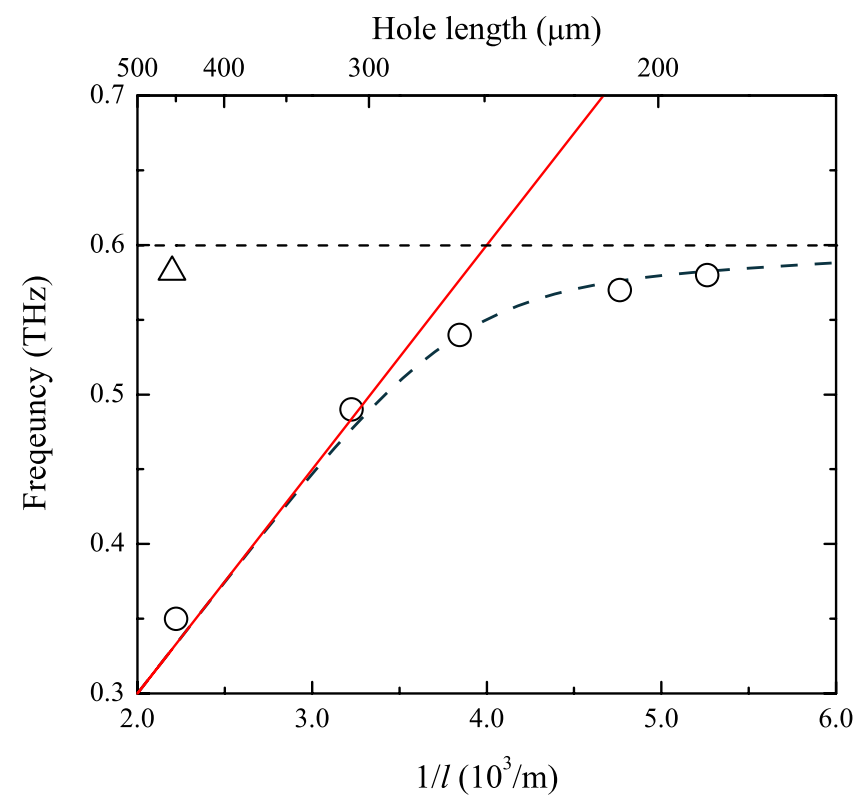

FIG. 2. (Color online) Transmission peak frequency (circles and triangles) as a function of $1 / l$. Resonant frequencies calculated from the theories of half-wavelength resonance (solid red line) and ESWs (black dotted line) are also shown for reference. The dashed line is the trajectory of the peak frequency; it serves as a guide to the eyes.

esting anticrossing behavior is observed when the frequencies of the two mechanisms are equal; this result indicates that critical coupling occurs between the two mechanisms.

Generally, if such a coupling occurs between two mechanisms, the coupled modes split into two branches, namely, an upper branch and a lower branch. The dashed line shown in Fig. 2 corresponds to the lower branch. The upper branch, however, cannot be observed clearly. This might be due to the diffraction of the electromagnetic wave into higher-order diffraction modes. It should be noted that a small transmission peak is observed at $0.59 \mathrm{THz}$ for $l=450 \mu \mathrm{m}$ [see Fig. 1(a)]; it appears as a shoulder of the strong peak at $0.35 \mathrm{THz}$. The small peak can be observed more clearly in the simulated transmission spectrum [Fig. 1(b)], and it is plotted in Fig. 2 (triangle). It mainly originated from ESWs and corresponds to a part of the upper branch.

In order to investigate the transition of the two mechanisms in more detail, we study the finite effect of the incident-beam diameter on the transmission spectrum. We need a large illuminated area for achieving strong ESWs, ${ }^{23}$ because it is caused by coherent interference of electromagnetic waves scattered by many holes. In contrast, such a large area is not required to produce localized resonance, because it is expected to be independent of the size of the illuminated area. Figures 3(a)-3(c) show the transmission spectra of three MHAs measured at $l=210,260$, and $410 \mu \mathrm{m}$, respectively. The values of the incident-beam diameter $d$ are 10 $\mathrm{mm}$ (solid line) and $1 \mathrm{~mm}$ (open circles). Since the size of all the samples is more than $10 \times 10 \mathrm{~mm}^{2}$, we can directly compare the transmittances obtained at the two values of $d$. Figure 3(a) shows that when $l=210 \mu \mathrm{m}$, the transmittance strongly depends on $d$. The transmittance at the peak frequency for $d=1 \mathrm{~mm}$ is significantly less than that for $d$ 


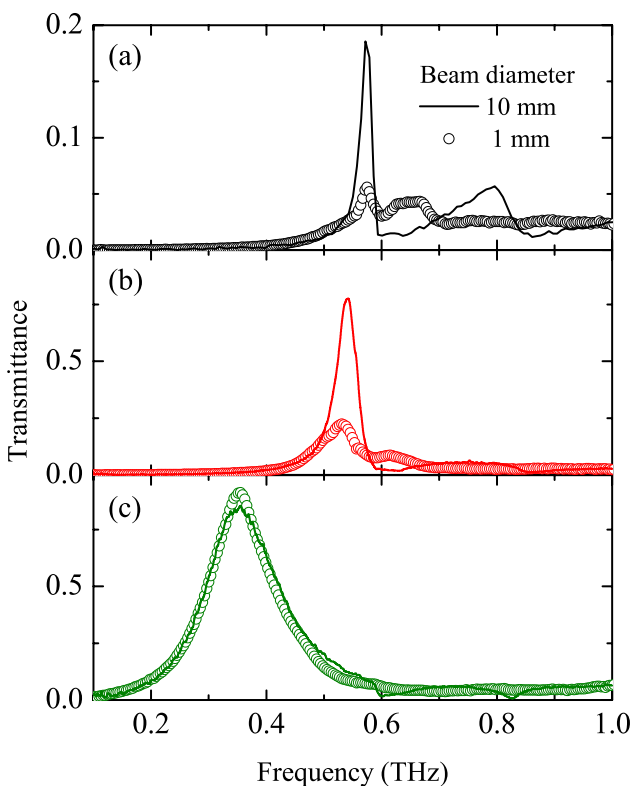

FIG. 3. (Color online) Transmission spectra of metal hole arrays measured at incident-beam diameters of $10 \mathrm{~mm}$ (solid line) and 1 $\mathrm{mm}$ (circle) and $l$ values of (a) 210, (b) 260, and (c) $410 \mu \mathrm{m}$.

$=10 \mathrm{~mm}$; this result indicates that ESWs are dominant in the sample produced at $l=210 \mu \mathrm{m}$. The dependence of transmittance on $d$ decreases with increasing $l$. At $l=410 \mu \mathrm{m}$, the difference between the transmission peaks for $d=10$ and 1 $\mathrm{mm}$ is very small [Fig. 3(c)]. This implies that localized resonance is the dominant mechanism in MHAs whose $l$ values are greater than the half-period. These results are in good agreement with those predicted from Fig. 2, as discussed in the previous paragraph.

The relatively high transmission peak appears in the spectra, regardless of which mechanism is dominant, and the peak frequency shifts continuously as the dominant mechanism changes from ESWs to localized resonance. This is a very interesting result because the difference between the two mechanisms cannot be determined only from the transmission spectra. It appears that the mechanism underlying resonant transmission depends on $l$. Therefore, the characteristics of the two mechanisms should be different, and they should exhibit different dependences on some physical parameters. The dependence of transmittance on $d$, as shown in Fig. 3, is one of the indicators of the difference between two mechanisms.

We also investigate the dependence of transmittance on the surface condition of the MHAs. When ESWs is the dominant mechanism, the electromagnetic field is strongly localized in the vicinity of the metal surface. In this case, the peak frequency depends on the permittivity of the dielectric attached to the metal surface. If the thickness of the dielectric is assumed to be infinite, the frequency of ESWs can be expressed as ${ }^{2}$

$$
f_{\mathrm{SW}}=\left|\mathbf{k}_{\mathrm{in}}+\mathbf{G}\right| \frac{c}{2 \pi}\left(\frac{\varepsilon_{m}+\varepsilon_{d}}{\varepsilon_{m} \varepsilon_{d}}\right)^{1 / 2},
$$

where $\mathbf{k}_{\text {in }}$ is the in-plane wave vector of the incident electromagnetic wave, $\mathbf{G}$ is the reciprocal-lattice vector, and $\varepsilon_{m}$ and

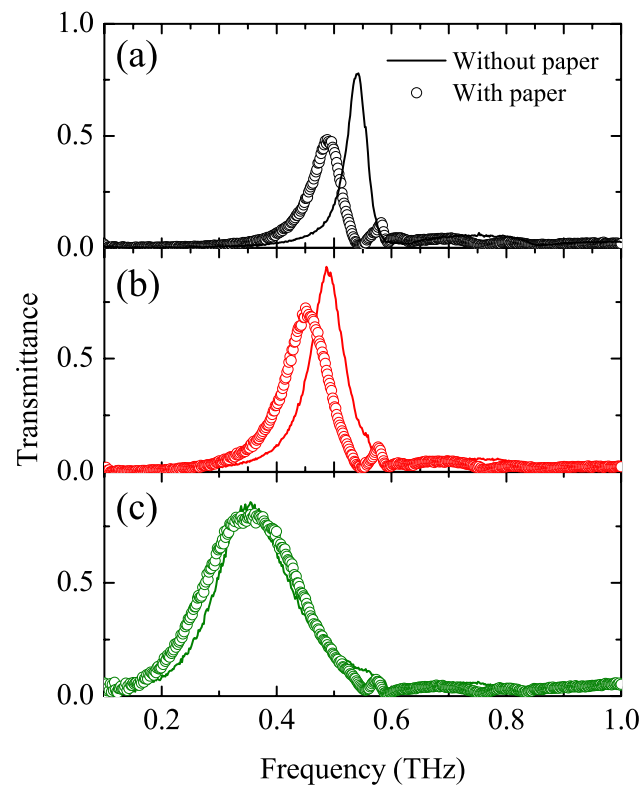

FIG. 4. (Color online) Transmission spectra of metal hole arrays with (circles) and without (solid line) the paper. These spectra were measured at $l$ values of (a) 260, (b) 310, and (c) $410 \mu \mathrm{m}$.

$\varepsilon_{d}$ are the dielectric constants of the metal and dielectric, respectively. However, when localized resonance is dominant, the peak frequency is expected to be almost independent of the surface condition of the MHAs. We measured transmission spectra of MHAs whose surfaces were covered with paper with a thickness of $100 \mu \mathrm{m}\left(\varepsilon_{\text {paper }}=2.2\right.$ at 0.5 $\mathrm{THz}$ ). The paper was used to change the permittivity above the metal surface. Figures 4(a)-4(c) show the transmission spectra of the samples (circles) covered with the paper; the hole lengths of these samples were $l=260,310$, and $450 \mu \mathrm{m}$, respectively. The transmission spectra of samples without paper are also shown in each figure for reference (solid line). In Fig. 4(a), the peak frequency of the spectra of samples covered with the paper shows a redshift of $\Delta f$ $=0.05 \mathrm{THz}$ in comparison with the peak frequency of samples without paper. This result is in qualitative agreement with that expected from Eq. (1). The value of $\Delta f$ decreases as $l$ increases [see Fig. 4(b)]. Furthermore, when localized resonance is the dominant resonant mechanism [Fig. 4(c)], the transmission peaks of the two samples with and without paper almost coincide with each other. This shows that resonant transmission is independent of the surface condition of the metal.

The discrepancy in the transmission properties that depend on the surface condition is due to different spatial distributions of the electromagnetic field for the two mechanisms. Bravo-Abad et al. ${ }^{12}$ investigated the hole size dependence of the transmission properties of square hole arrays and found that electric field distributions in the vicinity of the metal surface strongly depend on the hole size. When the hole length is less than the half-period of the square lattice structure, the electric field is concentrated on the metal surface. However, when the hole length is greater than the half-period, the electric field is concentrated inside the holes. Thus, as the hole length increases, the dependence of the 
transmission properties on the surface condition tends to decrease. Consequently, the peak frequency becomes independent of any change in the surface condition, as indicated by our results [Fig. 4(c)].

In summary, we have investigated the transition of the dominant resonant transmission mechanism in metal hole arrays by varying the length of the rectangular holes. As the hole length increases, the dominant mechanism changes continuously from ESWs to localized resonance. We have also demonstrated that the surface effect in resonant transmission strongly depends on the dominant mechanism. This depen- dence shows that the transmission characteristics during localized resonance are different from those during ESWs. Our results provide important information for the development of high-sensitivity surface wave sensors using metal hole arrays.

This work was supported partially by a Grant-in-Aid for Promotion of Scientific Research from the Japan Society for the Promotion of Science (JSPS). F.M. is also supported by the Murata Science Foundation and the Konica Minoluta Imaging Science Foundation.

\footnotetext{
*miyamaru@shinshu-u.ac.jp

${ }^{1}$ R. Ulrich, Infrared Phys. 7, 37 (1967).

${ }^{2}$ H. Reather, Surface Plasmons on Smooth and Rough Surfaces and on Gratings (Springer-Verlag, Berlin, 1988).

${ }^{3}$ T. W. Ebbesen, L. J. Lezec, H. F. Ghaemi, T. Thio, and P. A. Wolff, Nature (London) 391, 667 (1998).

${ }^{4}$ M. Sarrazin and J.-P. Vigneron, Phys. Rev. B 71, 075404 (2005).

${ }^{5}$ F. J. García de Abajo, Rev. Mod. Phys. 79, 1267 (2007).

${ }^{6}$ F. Miyamaru, M. Tanaka, and M. Hangyo, Phys. Rev. B 74, 115117 (2006).

${ }^{7}$ K. J. Klein Koerkamp, S. Enoch, F. B. Segerink, N. F. van Hulst, and L. Kuipers, Phys. Rev. Lett. 92, 183901 (2004).

${ }^{8}$ J. Lee, M. Seo, D. Park, D. Kim, S. Jeoung, and C. Lienau, Opt. Express 14, 1253 (2006).

${ }^{9}$ Z. Ruan and M. Qiu, Phys. Rev. Lett. 96, 233901 (2006).

${ }^{10}$ J. Han, A. K. Azad, M. Gong, X. Lu, and W. Zhang, Appl. Phys. Lett. 91, 071122 (2007).

${ }^{11}$ J. W. Lee, M. A. Seo, D. H. Kang, K. S. Khim, D. S. Kim, and S. C. Jeoung, Phys. Rev. Lett. 99, 137401 (2007).

${ }^{12}$ J. Bravo-Abad, L. Martín-Moreno, F. J. García-Vidal, E. Hendry, J. Gómez Rivas, Phys. Rev. B 76, 241102(R) (2007).

${ }^{13}$ H. Cao and A. Nahata, Opt. Express 12, 3664 (2004).
}

${ }^{14}$ K. L. van der Molen, K. J. Klein Koerkamp, S. Enoch, F. B. Segerink, N. F. van Hulst, and L. Kuipers, Phys. Rev. B 72, 045421 (2005).

${ }^{15}$ J. A. H. van Nieuwstadt, M. Sandtke, R. H. Harmsen, F. B. Segerink, J. C. Prangsma, S. Enoch, and L. Kuipers, Phys. Rev. Lett. 97, 146102 (2006).

${ }^{16}$ E. Popov, M. Neviere, J. Wenger, P.-F. Lenne, H. Rigneault, P. Chaumet, N. Bonod, J. Dintinger, and T. Ebbesen, J. Opt. Soc. Am. A Opt. Image Sci. Vis 23, 2342 (2006).

${ }^{17}$ T. K. Wu, Frequency Selective Surface and Grid Array (Wiley, New York, 1995).

${ }^{18}$ F. Miyamaru, S. Hayashi, C. Otani, K. Kawase, Y. Ogawa, H. Yoshida, and E. Kato, Opt. Lett. 31, 1118 (2006).

${ }^{19}$ F. Miyamaru, M. Takeda, T. Suzuki, and C. Otani, Opt. Express 15, 14804 (2007).

${ }^{20}$ F. Miyamaru, T. Kondo, T. Nagashima, and M. Hangyo, Appl. Phys. Lett. 82, 2568 (2003).

${ }^{21}$ A. P. Hibbins, M. J. Lockyear, I. R. Hooper, and J. R. Sambles, Phys. Rev. Lett. 96, 073904 (2006).

${ }^{22}$ J. Lee, M. Seo, D. Park, S. Jeoung, and Q. Park, Opt. Express 14, 12637 (2006).

${ }^{23}$ F. Miyamaru and M. Hangyo, Appl. Phys. Lett. 84, 2742 (2004). 